interner Vorstellungen abhängt. Gerade angesichts der Konfliktlinien, die quer zu den klassischen (Soll-)Bruchstellen laufen, wäre eine vertiefende Betrachtung der innerparlamentarischen Aushandlungsprozesse und Mechanismen, die zur Durchsetzung einer bestimmten Position führen, aufschlussreich. So wirft die Vielschichtigkeit sowohl der Legitimitätsmuster als auch, und damit korrespondierend, der Positionierung des Bundestags die Frage auf, wie diese in kollektives Handeln des Bundestags übersetzt werden: Welche Position setzt sich wann und wie durch? Ob und unter welchen Bedingungen hemmen sich konfligierende Deutungen gegenseitig und tragen zu der beklagten Inaktivität des Bundestags bei?

Insgesamt hat, viertens, die Verlagerung auf die Mikroebene und die Verwendung diskursanalytischer Werkzeuge eröffnet, dass ein plausibler Zusammenhang zwischen der Positionierung des Bundestags und Legitimitätsmustern besteht. Durch die in die Tiefe gehende Betrachtung wurde deutlich, dass es nicht ausreicht, eine Analyse des Legitimitätsdenkens auf idealtypische Finalitätsvorstellungen oder reduzierte Narrative zu begrenzen. Naturgemäß konnte lediglich ein Ausschnitt der Realität (sowohl der Akteure als auch in zeitlicher Perspektive) betrachtet werden, so dass die gezogenen Schlüsse tentativ bleiben. Eine Ausweitung des zeitlichen Horizonts und die Erhebung und Analyse vergleichbarer Daten in diesem Rahmen ist daher anzuregen. Vielversprechend scheinen hier die zunehmenden Bemühungen der Critical Discourse Analysis zu sein, verstärkt dem Dreieck Diskurs-Kognition-Gesellschaft/Umwelt und dessen Dynamik Rechnung zu tragen ${ }^{59}$, womit sich eine Verknüpfung linguistischer und politikwissenschaftlicher Forschung anbietet.

59 Vgl. Teun van Dijk, Critical Discourse Studies: A Sociocognitive Approach, in: Ruth Wodak I Michael Meyer (Hrsg.), Methods of Critical Discourse Analysis, London 2009, S. 62 - 86; Ruth Wodak, ,Doing Europe': The Discursive Construction of European Identities, in: Richard Mole (Hrsg.), Discursive Constructions of Identity in European Politics, Houndmills 2007, S. 70 - 94.

\title{
Subsidiarität und Mitsprache. Nationale Parlamente nach Lissabon
}

\author{
Aron Buzogány und Andrej Stuchlik
}

Innerhalb kurzer Zeit haben die formalen Instrumente der Subsidiaritätskontrolle, insbesondere der parlamentarische Frühwarnmechanismus des Lissabon-Vertrages ${ }^{1}$, bei Praktikern wie in der Wissenschaft viel Beachtung erfahren. Grund dieser Aufmerksamkeit ist die damit verbundene Aussicht, mittels neuer ex-ante Kontrollverfahren die Rolle der nationa-

1 Vertrag von Lissabon zur Änderung des Vertrags über die Europäische Union und des Vertrags zur Gründung der Europäischen Gemeinschaft vom 13. Dezember 2007, ABl. C306 vom 17. Dezember 2007 (nachfolgend Lissabon-Vertrag), in Kraft getreten am 1. Dezember 2009. Siehe auch Protokoll über die Rolle der nationalen Parlamente in der Europäischen Union, ABl. C 306/148 (nachfolgend Parlamente-Protokoll); Protokoll über die Anwendung der Grundsätze der Subsidiarität und der Verhältnismäßigkeit, ABl. C306/150 (nachfolgend Subsidiaritäts-Protokoll). 
len und regionalen Parlamente, die gewöhnlich als „Integrationsverlierer“ erachtet wurden², wieder zu stärken. Zwei Jahre nach Inkrafttreten drängt sich die Frage auf, inwiefern diese formalen Neuerungen und die damit verbundenen Demokratisierungsbemühungen auch tatsächlich den Einfluss der gesetzgebenden Repräsentativkörperschaften der Mitgliedstaaten befördert haben.

Zumeist kreisen Debatten zu den Auswirkungen fortschreitender Integration auf demokratische Legitimität und innerstaatliche Effektivität um die Frage, ob und wie Parlamente es verstanden haben „zurückzuschlagen “3 und über welche innerstaatlichen europapolitischen Kontrollmechanismen und Routinen sie verfügen. ${ }^{4}$ Entsprechend zählten und zählen Gestalt und Wahl dieser parlamentarischen Mitspracherechte zum Gegenstand einer reichhaltigen Forschungsliteratur. ${ }^{5}$ Je nach Fragestellung hängen Ausbau und Nutzung dieser „scrutiny rights“ von folgenden Faktoren ab: Verhältnis von Regierung und Ministerialbürokratie, Existenz von Minderheitsregierungen, Politischer Kultur, parteipolitischer und tatsächlicher Euroskeptizismus, relative Stärke von Parlamentsausschüssen, und zwar ganz unabhängig von europapolitischen Aufgaben.

Dabei steht die These der so genannten Entparlamentarisierung im Kern der parlamentsbasierten EU-Forschung der letzten beiden Jahrzehnte. ${ }^{6}$ Demzufolge ist der europäische Einigungsprozess ein Grund, wenn auch möglicherweise nur einer von vielen, für den Bedeutungsverlust von Parlamenten in den EU-Mitgliedstaaten. Die europäische Integration habe sowohl zu verfassungsrechtlichen als auch zu politischen Entwicklungen beigetragen, die die Machtbasis der legislativen Organe verringert hätten. Der Souveränitätsverlust sei in Kauf genommen worden für die Aussicht auf größeren (potenziellen) Nutzen durch die Zugehörigkeit zum regionalen Wirtschaftsraum. Ein großer Anteil der innerstaatlichen Gesetze gehe infolgedessen auf europäisches Recht zurück, wenngleich der Mythos der „80 Prozent" ${ }^{\text {"üngst relativiert worden ist. }}{ }^{7}$

2 Für eine Zusammenfassung der Debatte siehe Klaus H. Goetz / Jan-Hinrik Meyer-Sahling, The Europeanization of National Political Systems: Parliaments and Executives, in: Living Reviews in European Governance, 3. Jg. (2008), H. 2.

3 Vgl. insbesondere Tapio Raunio / Simon Hix, Backbenchers Learn to Fight Back: European Integration and Parliamentary Government, in: West European Politics, 23. Jg. (2000), H. 4, S. 142 $-168$.

4 Stellvertretend für viele Katrin Auel / Arthur Benz, The Europeanisation of Parliamentary Democracy, London 2005.

5 Vgl. Andreas Maurer, Post-2004 Elements for Comparative Analysis of National Parliamentary Participation in EU Affairs, in: Jürgen Dieringer / Enikö Györi / ders. (Hrsg.), Europäische Entscheidungen kontrollieren! Nationale Parlamente im Ost-West-Vergleich, Dresden 2005, S. 49 89; Tapio Raunio, National Parliaments and European Integration: What We Know and Agenda for Future Research, in: The Journal of Legislative Studies, 15. Jg. (2009), H. 4, S. 317 - 334.

6 Vgl. Klaus H. Goetz / Jan-Hinrik Meyer-Sahling, a.a.O. (Fn. 2), S. 6 ff.

7 Zur schwierigen Einschätzung tatsächlich EU-induzierter Gesetzgebung siehe Annette Elisabeth Töller, Mythen und Methoden. Zur Messung der Europäisierung der Gesetzgebung des Deutschen Bundestages jenseits des 80-Prozent-Mythos, in: ZParl, 39. Jg. (2008), H. 1, S. 3 - 17; dies., Measuring and Comparing the Europeanization of National Legislation: A Research Note, in: Journal of Common Market Studies, 48. Jg. (2010), H. 2, S. 417 - 444; Jørgen Grønnegaard Christensen, EU Legislation and National Regulation: Uncertain Steps towards a European Public Policy, in: Public Administration, 88. Jg. (2010), H. 1, S. 3 - 17. Kritisch dazu Sven Hölscheidt I Tilman Hoppe, Der Mythos vom „europäischen Impuls“ in der deutschen Gesetzgebungsstatistik, in: ZParl, 41. Jg. (2010), H. 3, S. 543 - 551. 
Allerdings ist mit Entparlamentarisierung lediglich eine Seite der Medaille beschrieben. Ihr wurde in der Vergangenheit von nationalen Gesetzgebungsorganen in vielfältiger Art und Weise mit Maßnahmen der Reparlamentarisierung begegnet, um den Nachteil gegenüber der Exekutive zu verringern. ${ }^{8}$ Bereits mit dem Inkrafttreten der Römischen Verträge Ende der 1950er Jahre haben nationale Parlamente Europaausschüsse eingesetzt, um die Brüsseler Aktivitäten ihrer Regierungen zu überwachen. Diese Bemühungen haben seit dem Maastrichter Vertrag von 1993 zugenommen: Die Ausweitung supranationaler Kompetenzen wurde flankiert von einer Zunahme an Mitsprache- und Kontrollbefugnissen seitens mitgliedstaatlicher Parlamente. Waren beispielsweise Abgeordnete bis dato zumeist auf die großzügige Bereitstellung an Informationen durch Regierungen angewiesen, so erarbeiteten sie sich fortan formelle wie informelle Zugänge. ${ }^{9}$ Eine der größten Herausforderungen lag im Bewusstsein, stets "einen Schritt zu spät” zu kommen ${ }^{10}$, wodurch die gestaltende Rolle („policy-shaping“) der gesetzgebenden Gewalt zu einer passiven („policy-taking“) reduziert wurde. ${ }^{11}$ Um dieser Entwicklung etwas entgegensetzen zu können, haben nationale Parlamente ihre eigenen Büros in Brüssel ${ }^{12}$ eröffnet und darüber hinaus enge Beziehungen zu Abgeordneten des Europäischen Parlaments geknüpft. ${ }^{13}$ Die Zusammenarbeit mit anderen Gesetzgebern hat zu einem interparlamentarischen Forum geführt, der sogenannten COSAC-Konferenz ${ }^{14}$, die die Parlamentarier der jeweiligen Europaausschüsse zusammenbringt. ${ }^{15}$

Wenngleich langsam, so haben diese Aktivitäten doch allmählich zu einer stärkeren Wahrnehmung der nationalen Parlamente im Politikprozess geführt, sowohl seitens des Europäischen Parlaments als auch seitens der Kommission. ${ }^{16}$ Im Windschatten der anhaltenden Kritik am Demokratiedefizit der EU fand die Bedeutung der nationalen Kammern ihren Weg in das europäische Vertragswerk. Ihre im Maastrichter Vertrag 1993 noch zögerlich formulierte Bedeutung wurde durch das in Amsterdam 1997 beschlossene und im Lissaboner Vertrag 2007 ergänzte „Protokoll über die Rolle der nationalen Parlamente“ gestärkt und ausgebaut. Die wesentlichen Fragen erscheinen dabei, inwieweit mitgliedstaatliche Parla-

8 Vgl. John O'Brennan / Tapio Raunio, Introduction: Deparliamentarization and European Integration, in: dies. (Hrsg.), National Parliaments within the Enlarged European Union. From 'Victims' of Integration to Competitive Actors, London 2007, S. 1 - 26.

9 Vgl. die Beiträge von Birgit Daiber, Hinrich Schröder und Richard Wichmann zum Thema in diesem Heft.

10 Vgl. Tapio Raunio, Always One Step Behind? National Legislatures and the European Union, in: Government and Opposition, 34. Jg. (1999), H. 2, S. $180-202$.

11 Vgl. Tanja A. Börzel / Carina Sprungk, Undermining Democratic Governance in the Member States? The Europeanization of National Decision-making, in: Ronald Holzhacker / Erik Albaek (Hrsg.), Democratic Governance and European Integration: Linking Societal and State Processes of Democracy, Aldershot 2007, S. 113 - 136.

12 Jedoch erst recht spät mit dem Deutschen Bundestag als Vorreiter seit 2007.

13 Vgl. Karlheinz Neunreither, The European Parliament and National Parliaments: Conflict or Cooperation?, in: The Journal of Legislative Studies, 11. Jg. (2005), H. 3/4, S. 466 - 489.

14 Conference of Community and European Affairs Committees / Conférence des Organes Spécialisés dans les Affaires Communautaires et Européennes des Parlements de l'Union Européenne, eingesetzt im Jahr 1989, http://www.cosac.eu/en/ (Abruf am 16. Mai 2012).

15 Vgl. Christina Bengtson, Interparliamentary Cooperation within Europe, in: John O'Brennan I Tapio Raunio (Hrsg.), a.a.O. (Fn. 8), S. 46 - 63, S. 50 ff.

16 So auch die Ausführungen eines Kommissionsbeamten, der die wachsende Bedeutung nationaler Parlamente hervorhob (eigenes Interview). 
mente an Einfluss de facto hinzugewonnen haben und ihn zudem auch zu nutzen wissen. Optimistische Deutungen über die Auswirkung der Änderungen im Lissaboner Vertrag lassen hoffen, dass sich nationale Parlamente dadurch als relevante Akteure im europäischen Mehr-Ebenen-System etablieren können. ${ }^{17}$ Ein erster wichtiger Aspekt ist dabei die zunehmende intraparlamentarische Zusammenarbeit auf europäischer Ebene. So unterstreichen Analysen die erhöhten Anreize für konzertiertes Vorgehen ${ }^{18}$, die durchaus zur Schaffung neuer, wenn auch informeller Institutionen wie einer „Virtuellen Dritten Kammer“ 19 oder einem „Conseil d'Etat“20 nationaler Parlamente führen könnten. Zweitens können die neugeschaffenen Instrumente indirekt dazu beitragen, die europarechtliche Dimension im parlamentarischen Alltagsgeschäft bewusster werden zu lassen. Insofern würde auch die Kritik zahlreicher Abgeordneter entkräftet, sie würden im EU-Entscheidungsprozess nicht hinreichend berücksichtigt oder zum „Stimmvieh“ reduziert sein. ${ }^{21}$ Mittelbar dürfte eine solche „EU-isierung“ der Öffentlichkeit zu einer größeren Legitimität der Union insgesamt führen.

Andere haben dagegen bereits früh die Sorge geäußert, dass die neuen Mitwirkungsrechte so komplex ausgefallen seien, dass verhältnismäßig wenig von ihnen zu erwarten sei. ${ }^{22}$ Ein Grund dafür sind die hohen Mehrheitsquoren, die erforderlich sind, um die kollektive Stimme der nationalen Kammern im ordentlichen Gesetzgebungsverfahren (Art. 294 AEUV) erheben zu können, so dass bereits der Versuch, diese überhaupt zu erreichen, unattraktiv ausfallen könnte.

Die Ausstattung nationaler Parlamente mit größeren Kontrollbefugnissen in der Europapolitik birgt zudem die Gefahr, diese in mehrfacher Hinsicht zu überfordern: Sie müssen nicht allein institutionelle Anpassungen vornehmen, um die steigende Flut an Zuleitungen beherrschbar zu machen. ${ }^{23}$ Insbesondere ist den vom Vertrag von Lissabon gewünschten Eingriffsbefugnissen der mitgliedstaatlichen Gesetzgeber eine strukturelle Schranke gesetzt, die sich nicht de lege ferenda aufheben lässt, denn natürlich stützt in parlamentarischen Regierungssystemen (außer bei seltenen Minderheitsregierungen) die Mehrheit das Regierungshandeln und steuert somit mittelbar das Entscheidungskalkül im Ministerrat.

Insofern stellt sich stets die Frage, ob nicht durch verstärkte parlamentarische Aufsicht die Verhandlungsposition einer Regierung auf der europäischen Bühne geschwächt würde, indem diese gezwungen wird, sich mit einem widerspenstigen Parlament abstimmen zu müssen. Während dies unter dem Gesichtspunkt größerer Transparenz zwar gute Nachrichten sind, führt es wohl nicht zu höherer Effizienz. ${ }^{24}$ Noch negativer gefasst, könnten Regierungen dazu neigen, sich hinter ihren nationalen Gesetzgebungskörperschaften zu „ver-

17 Vgl. Gavin Barrett, National Parliaments and the European Union, Dublin 2008.

18 Vgl. Davor Jančić, A New Organ of the European Union: National Parliaments Jointly, Federal Trust for Education and Research, London 2008.

19 Ian Cooper, A Virtual Third Chamber for the European Union? National Parliaments after the Treaty of Lisbon, Oslo 2011.

20 Philip Kiiver, The Early Warning System for the Principle of Subsidiarity: The National Parliament as a Conseil d'Etat for Europe!, in: European Law Review, 36. Jg. (2010), H. 1, S. 98 - 108.

21 Vgl. Stefan Tillmann, Stimmvieh, in: Financial Times Deutschland vom 21. April 2011, S. 25 f.

22 Vgl. Ricardo Passos, Recent Developments Concerning the Role of National Parliaments in the European Union, in: ERA Forum, 9. Jg. (2008), H. 1, S. 5 - 40.

23 Vgl. Janis A. Emmanouilidis / Corina Stratulat, Implementing Lisbon: Narrowing the EU's 'Democratic Deficit'?, European Policy Centre, Brüssel 2010.

24 Vgl. ebenda. 
stecken“, um missliebige EU-Vorstöße mit Verweis auf diese „scrutiny reserve“ zu blockieren. ${ }^{25}$ Im europäischen Politikalltag können strategische Hinweise auf solcherlei parlamentarische Kontrolle erheblichen Einfluss im Ausschuss der Ständigen Vertreter sowie in den Arbeitsgruppen des Rates entfalten.

Vor diesem Hintergrund ist es lohnend, die Spielräume, Kapazitäten und Anreizstrukturen nationaler Parlamente in ihrem Bemühen zu untersuchen, ihre neugewonnen Kompetenzen einzusetzen. Dabei wird es vor allem um die parlamentarischen Kontrollmechanismen gehen, die Neuerungen des Lissaboner Vertragswerkes sowie die sehr unterschiedlichen Anpassungsleistungen und intra- wie interparlamentarischen Kooperationsstrukturen. ${ }^{26}$

\section{Parlamentarische Kontrolle europäischer Politik in den Mitgliedstaaten}

\subsection{Status quo ante}

Die wissenschaftliche Beschäftigung mit parlamentarischem Einfluss und Kontrolle von europäischer Politik hat sich in überwiegendem Maße auf Europaausschüsse und deren institutionelle Verankerung konzentriert. ${ }^{27}$ Im Verlauf der letzten 20 Jahre hat nicht allein ihre Zahl zugenommen, sondern ebenfalls ihr politisches Gewicht. Ausgehend von den „best practices“ Dänemarks, Österreichs, Schwedens oder Finnlands, denen besonders weitreichende parlamentarische Kontrollbefugnisse attestiert werden, haben die jüngeren EUMitgliedstaaten Mittel- und Osteuropas versucht gleichzuziehen und haben einige dieser institutionellen Blaupausen übernommen. ${ }^{28}$

Das Monitoring europäischer Politik im nationalen legislativen Kontext variiert in vielerlei Hinsicht: in Bezug auf Zeitpunkt und Umfang von zugeleiteten Informationen seitens der Regierung, der „Bindekraft“ parlamentarischer Mandate für das Abstimmungsverhalten der nationalen Regierung im Rat sowie der Berücksichtigung weiterer Fachausschüsse bei der parlamentarischen Beschlussfassung. Hinzu kommen wichtige Unterschiede etwa bei der Tagungshäufigkeit der Europa-Ausschüsse, der Einbeziehung einzelner Abgeordneter

25 Gleichwohl wäre das keine ganz neue Entwicklung: So brachten Regierungen etwa während der 45 Sitzungen des AStV I im Jahre 2008 insgesamt 46 Parlamentsvorbehalte ein.

26 Es wurden unterschiedliche Informationsquellen herangezogen: Angaben nationaler Parlamente in Umfragen, COSAC-Berichte sowie Experteninterviews mit Abgeordneten und Mitgliedern von Parlaments- und Ministerialverwaltungen. Die meisten dieser Interviews wurden im Rahmen eines Forschungsprojektes am Deutschen Forschungsinstitut für Öffentliche Verwaltung Speyer erhoben. Das Projekt untersucht die Interaktionen und wechselseitigen Rollenwahrnehmungen von Abgeordneten und Spitzenbeamten, die in unterschiedlich europäisierten Fachpolitiken engagiert sind. Siehe http://www.foev-speyer.de/Rolleneuropa/inhalte/01_home.asp (Abruf am 16. Mai 2012).

27 Vgl. Andreas Maurer / Wolfgang Wessels, National Parliaments on their Ways to Europe: Losers or Latecomers, Baden-Baden 2001; Katrin Auel / Arthur Benz, a.a.O. (Fn. 4).

28 Vgl. Jürgen Dieringer / Andrej Stuchlik, Die Europäisierung der Parlamentsarbeit in Ungarn und der Tschechischen Republik, in: Südosteuropa, 52. Jg. (2003), H. 7, S. 388 - 403; Aron Buzogány, Learning from the Best. Inter-Parliamentary Policy Learning and EU Parliamentary Scrutiny Design in the EU-10, in: Ben Crum / John E. Fossum (Hrsg.), Practices of Inter-Parliamentary Coordination in International Politics, Routledge (im Erscheinen). 
außerhalb des Europagremiums und bei der Frage von Finanz-und Personalausstattung. Dennoch lassen sich im Wesentlichen zwei Gruppen unterteilen ${ }^{29}$ : Die erste, das so genannte dokumentenbasierte Modell, betont die prozedurale Komponente parlamentarischer Kontrolle. Das bedeutet, dass Europaausschüsse sämtliche zugeleiteten EU-Dokumente sichten. Die darauffolgende Vorauswahl und Priorisierung übernimmt im Regelfall ein Sekretariat. Bei dieser institutionellen Lösung stehen Beratungen und Informationsverarbeitung im Zentrum. So können Europaausschüsse etwa Sachverständige zu Anhörungen laden, um ihren internen Willensbildungsprozess zu stärken. Wesentlich ist dabei, dass nicht versucht wird, direkt über die Aktivität der eigenen Regierung in Brüssel Einfluss auszuüben. Dies heißt jedoch nicht, dass die EU-Ausschüsse die Regierungsposition nicht beeinflussen könnten. Vielmehr verschiebt sich der Schwerpunkt der Einflussnahme in parlamentarische Aussprachen und Expertengespräche.

Die zweite Gruppe parlamentarischer Kontrollstrukturen kreist um die Frage der angemessenen Weisungsbindung der Exekutive, das so genannte mandatierende Modell. So wie das britische Unterhaus geradezu ein idealtypischer Vertreter der ersten Gruppe ist, so gehört das dänische Parlament, der Folketing, mit der Erteilung von Verhandlungsmandaten der zweiten an. Diese Ausgestaltung hängt allerdings eng mit den Spezifika des dänischen Regierungssystems zusammen, in dem häufig Minderheitsregierungen gebildet werden. Daraus ergeben sich verhältnismäßig schwache Exekutiven, die sich einem relativ starken Parlament gegenüber sehen. Das Mandatierungsverfahren hat weniger die Informationskanäle als die tatsächliche Regierungsposition bei Ratsentscheidungen im Fokus. Aufgrund dessen wird die Agenda der Europaausschüsse eng an die Sitzungen des Ministerrates gekoppelt. Im parlamentarischen Alltag heißt dies, dass die Regierungen jeweils eine Woche vor Ratssitzungen mit klaren Stimmvorgaben ausgestattet werden. Allerdings variiert der aus parlamentarischen Mandatierungen resultierende exekutive Entscheidungsspielraum: Einige Mitgliedstaaten arbeiten systematisch mit Mandatierungen und können so Regierungsvertreter zum Umschwenken auf ihre Position bewegen. Andere verfügen zwar ebenfalls über die Möglichkeit, Voten zu binden, machen davon jedoch nur sehr unregelmäßig Gebrauch.

Der Vorteil der Unterteilung in diese zwei Gruppen liegt zunächst darin, Aufsichtskompetenzen formal systematisieren zu können. Gleichwohl existieren zum einen Mischformen und zum anderen ist damit, wie schon angedeutet, noch wenig über den tatsächlichen Gebrauch von Kontrollmöglichkeiten gesagt. Eine Vielzahl empirischer Arbeiten belegt - wenig überraschend -, dass formal schwach kontrollierende Kammern durchaus dann zu entschlossener Aufsicht und Einflussnahme gewillt sind, wenn die innenpolitische Bedeutung des jeweiligen Gegenstandes hinreichend groß ist. ${ }^{30}$

29 Vgl. Tapio Raunio, Holding Governments Accountable in European Affairs: Explaining CrossNational Variation, in: The Journal of Legislative Studies, 11. Jg. (2005), H. 4, S. 319 - 342.

30 Vgl. Paul Stephenson, Catching the Train to Europe: Executive Control of Policy Formulation inside Spain's Parliamentary European Union Affairs Committee, in: South European Society and Politics, 14. Jg. (2009), H. 3, S. 317 - 336; Christine Neuhold / Rik de Ruiter, Out of REACH? Parliamentary Control of EU Affairs in the Netherlands and the UK, in: The Journal of Legislative Studies, 16. Jg. (2010), H. 1, S. 57 - 72; Carina Sprungk, Ever More or Ever Better Scrutiny? Analysing the Conditions of Effective National Parliamentary Involvement in EU Affairs, in: European Integration online Papers (EIoP), 14. Jg. (2010), H. 2. 
Diese Unterschiede parlamentarischer Kontrollaktivität sind nicht zuletzt Ausdruck eines ebenfalls unterschiedlichen Umgangs mit Subsidiaritätsaspekten. ${ }^{31}$ In den meisten Mitgliedstaaten ist die Subsidiaritätsprüfung Teil des allgemeinen Zuleitungsverfahrens europäischer Gesetzesinitiativen. Einige wenige Kammern haben auch vor den Änderungen des LissabonVertrages spezifisch auf Verstöße gegen dieses Prinzip geprüft (italienischer Senat, litauischer Saemas), während einige jüngere Mitgliedstaaten keine spezifische EU-Prüfung vornahmen (Estland, Zypern, Polen und Rumänien). ${ }^{32}$

Zusammengenommen bedeutet dies, dass die Analyse und Bewertung der erweiterten Kontrollbefugnisse nationaler Parlamente in der EU dem Umstand Rechnung tragen muss, dass Akteure dabei verschiedenen Ansätzen des EU-Monitorings folgen und von unterschiedlichen Ausgangsbedingungen ausgehen. Wie im nächsten Abschnitt deutlich wird, spielen diese unterschiedlichen innerstaatlichen Arrangements eine erhebliche Rolle bei der Ausgestaltung und Nutzung der erweiterten Kompetenzen nach Lissabon.

\subsection{Stärkung parlamentarischer Kontrollrechte durch den Lissabon-Vertrag}

Nationale Kammern finden erstmals im Vertragswerk eine direkte Erwähnung sowie eine Aufwertung in zwei gesonderten Protokollen. Art. 5 Abs. 3 EUV weist ihnen innerhalb der Union explizit die Aufgabe zu, die Einhaltung der Subsidiarität zu gewährleisten ${ }^{33}$, während Art. 12 EUV ihre Rolle und Funktionen auflistet, unter anderem die Beurteilung der Umsetzungsgüte von Maßnahmen in Politikfeldern der Freiheit, der Sicherheit und des Rechts, der Bewertung der Tätigkeit von Europol und Eurojust sowie eine Beteiligung bei Vertragsänderungen. Das erste Protokoll beschreibt insbesondere Informationsrechte; das zweite legt die Verfahrensschritte dar, wonach die Prinzipien der Subsidiarität und Verhältnismäßigkeit geprüft werden sollen. ${ }^{34}$

Im Alltag bedeutet dies, dass die Kommission den nationalen Kammern ihre Gesetzesvorschläge unmittelbar zuleitet. Insofern stärkt der Vertragstext das Recht des einzelnen Abgeordneten im Bereich der Subsidiaritätskontrolle und erweitert die Zeitspanne, in der eine „begründete Stellungnahme“ (Subsidiaritätsrüge) vorgelegt werden kann, von ursprünglich sechs auf acht Wochen. Die Befassung mit einem Entwurf ${ }^{35}$ kann mit einer so genannten „gelben“ beziehungsweise „orangenen“ Karte geahndet werden. ${ }^{36}$ Auslöser für eine gelbe Karte ist die Nichtzustimmung von mindestens einem Drittel der nationalen Kammern (18 von

31 Vgl. Bureau of Research, Chancellery of the Sejm, Parliamentary Scrutiny of Compliance of EU Legislative Acts with the Principle of Subsidiarity, Konferenz: The Treaty of Lisbon - Treaty of European Parliaments, Warschau vom 22. bis 23. Februar 2010.

32 Vgl. ebenda, S. 1.

33 In seinem Urteil zum Lissabon-Vertrag betonte das Bundesverfassungsgericht explizit die „Integrationsverantwortung" des Deutschen Bundestages. Siehe BVerfG, 2 BvE 2/08 vom 30. Juni 2009, http://www.bundesverfassungsgericht.de/entscheidungen/es20090630_2bve000208.html (Abruf am 16. Mai 2012).

34 Vgl. Parlaments-Protokoll; Subsidiaritäts-Protokoll; Hans-Jürgen Papier, Zur Verantwortung der Landtage für die europäische Integration, in: ZParl, 41. Jg. (2010), H. 4, S. 903 - 908.

35 Entwürfe eines Rechtsetzungsaktes können von der Kommission, aber auch von anderen Organen und Institutionen der EU stammen (siehe Art. 3, Subsidiaritäts-Protokoll).

36 Art. 7 Abs. 2 und 7 Abs. 3 (Subsidiaritäts-Protokoll). 
54 Stimmen) ${ }^{37}$, aber die Kommission muss an ihrem Entwurf keine Änderungen vornehmen. Bei einer einfachen Mehrheit (27 von 54 Stimmen) der nationalen Kammern (orangene Karte) muss sie dagegen eine solche Entscheidung gegenüber dem Europäischen Parlament sowie dem Rat begründen. Diese müssen vor Ende der ersten Lesung zum Gesetzesvorschlag Stellung nehmen und können die Kommissionsinitiative mit 55 Prozent der Stimmen im Rat beziehungsweise einer einfachen Mehrheit im Europäischen Parlament ablehnen.

Eine weitere Stärkung ergibt sich aus der Tatsache, dass nun nationale Kammern im Rahmen der allgemeinen Brückenklausel das Recht erhalten, die Ausdehnung der qualifizierten Mehrheitsregel auf Bereiche vormaliger Einstimmigkeit im Rat blockieren zu können (Art. 48 Abs. 7 EUV). ${ }^{38}$ Erweiterte Kontrollbefugnisse wurden zudem der regionalen Ebene zuteil, so dass der Ausschuss der Regionen ebenfalls Subsidiaritätseinwände vorbringen kann. Darüber hinaus müssen Entwürfe von Gesetzgebungsakten künftig Folgenabschätzungen enthalten, sowohl hinsichtlich ihrer finanziellen Konsequenzen als auch ihrer administrativen Auswirkungen auf Regionen und Gemeinden. Allerdings ist die Rolle regionaler Parlamente bislang nicht hinreichend geklärt ${ }^{39}$, dabei ist die Lage insbesondere für deutsche Landtage virulent. Das Lissabon-Urteil des BVerfG hat zwar im Binnenverhältnis die Position der beiden gesetzgebenden Kammern gestärkt, doch die Landesparlamente suchen noch nach effektiven Mitteln erweiterter Mitsprache. Jüngst wurden Informationsflüsse und Kooperationsregeln zwischen den 16 Institutionen verbessert ${ }^{40}$ und werden weiterhin kontinuierlich überprüft.

\subsection{Anpassungen nationaler Parlamente an den Lissabon-Vertrag}

Die Anpassung der parlamentarischen Geschäftsordnungen der nationalen Parlamente an das EU-Vertragswerk weist deutliche Unterschiede auf. ${ }^{41}$ Einige Kammern betrachteten ihre Abstimmungsmechanismen als ausreichend, insbesondere in den mittel- und osteuropäischen Mitgliedstaaten, die in den Jahren zuvor die Lissabon-Vorgaben zum Teil bereits berücksichtigt hatten. ${ }^{42}$ In anderen Fällen reichte eine Änderung der Geschäftsordnung oder

37 Mindestens neun Mitgliedstaaten (18 Stimmen). Aber „[...] wenn es sich um einen Entwurf eines Gesetzgebungsakts auf der Grundlage des Art. 76 des Vertrags über die Arbeitsweise der Europäischen Union betreffend den Raum der Freiheit, der Sicherheit und des Rechts handelt", reicht ein Viertel der Stimmen, also mindestens sieben Mitgliedstaaten (14 Stimmen); vgl. ebenda.

38 Hinzu kommt auch ein Ablehnungsrecht bei der besonderen Brückenklausel nach Art. 81, Abs. 3 AEUV, bei Maßnahmen zum Familienrecht mit grenzüberschreitendem Bezug. Die Einspruchsfrist beträgt in beiden Fällen sechs Monate.

39 Dies ist besonders in jenen Mitgliedstaaten relevant, die mit diesen Akteuren kooperieren: Österreich, Belgien, Deutschland, Italien, Portugal, Spanien und Großbritannien.

40 Vgl. zum Beispiel Landtag Brandenburg, Sachstandsbericht über die Aktivitäten der deutschen Länderparlamente in Fragen der Subsidiaritätskontrolle sowie über den Diskussionsstand auf der Ebene der Landtagspräsidenten, Drs. 4/4015, Potsdam vom 23. Januar 2007; Thüringer Landtag, Wolfsburger Erklärung der Konferenz der Präsidentinnen und Präsidenten der deutschen und österreichischen Landesparlamente sowie des Tiroler Landtags, Drs. 5/2872, Erfurt vom 7. Juni 2011.

41 Vgl. COSAC, Annex to the Thirteenth Bi-annual Report on Developments in European Union Procedures and Practices Relevant to Parliamentary Scrutiny: Replies of National Parliaments and the European Parliament. Prepared by the COSAC Secretariat and Presented to: XLIII Conference of Community and European Affairs Committees of Parliaments of the European Union, Madrid vom 31. Mai bis 1. Juni 2010.

42 Vgl. ebenda, S. 12. 
der Verfahrensabläufe (die belgische Chambre des représentants, die tschechische Poslanecká sněmovna, die finnische Eduskunta, die französische Assemblée nationale, das griechische Vouli ton Ellinon, die niederländische Eerste Kamer und Tweede Kamer, der schwedische Riksdag sowie das britische House of Lords ${ }^{43}$ ), teils wurden allerdings noch zusätzlich gesetzliche Anpassungen beschlossen (für beide Kammern in Deutschland, Tschechien und den Niederlanden, in Finnland, Frankreich, Griechenland, Schweden und Slowenien sowie für das britische House of Lords). Zu Verfassungsänderungen kam es darüber hinaus im deutschen, französischen und österreichischen (Lissabon-Begleitnovelle) Fall.

Dabei zeichneten sich wiederum im Wesentlichen zwei Ansätze ab, wie die parlamentarische Mitsteuerung europäischer Politik zu vollziehen sei: Im ersten wird diese Aufsichtsfunktion explizit den Europaausschüssen zugewiesen (zum Beispiel House of Lords, Zypern, Slowakei). Falls notwendig, beziehen diese Ausschüsse die Arbeit weiterer Fachausschüsse mit ein (zum Beispiel die niederländischen Vaste commissie voor Europese Zaken), dem Querschnittsgremium obliegt jedoch die Federführung.

Im zweiten Ansatz werden dagegen die einzelnen Fachausschüsse zulasten der EU-Ausschüsse in ihrer Aufsichtsfunktion gegenüber der Regierung gestärkt und die Verantwortung sektoral verteilt. ${ }^{44}$ Beiden Ansätzen gemeinsam ist die zentrale Bedeutung des Timings für eine effektive Kontrolle. Obwohl effektive ex-ante Kontrolle zu einem früheren Zeitpunkt ansetzen müsste, starten die Verfahren erst mit der förmlichen Zuleitung der Dokumente durch die Europäische Union. Die Frage, ob Ressourcen der Regierungen und der Parlamentsverwaltungen bei der Sichtung und Priorisierung der eingegangenen Entwürfe angezapft werden können, ist unterschiedlich geregelt; in manchen Mitgliedstaaten sind die Exekutiven dazu verpflichtet (Deutschland, Bulgarien, Slowenien, Portugal, Großbritannien, Finnland), in anderen können sie auf Anfrage hinzugezogen werden (Frankreich, Zypern, Niederlande, Schweden).

Allerdings legen die nationalen Unterschiede bei der Ausgestaltung der Subsidiaritätskontrolle den politischen und damit interpretierbaren Gehalt des Begriffs „Subsidiarität" 45 offen (siehe oben). Diese politische Norm wird von mitgliedstaatlichen Gesetzgebern (und Judikativen) recht unterschiedlich ausgelegt. So variiert auch der Sprachgebrauch auf der Ebene der einzelnen Abgeordneten je nach ihrer nationalen, regionalen oder europäischen Verankerung. Wie eine, allerdings recht begrenzte, Untersuchung ergab, offenbaren sich dabei nur „krude Vermutungen“ über den Wesensgehalt dieses Begriffes. ${ }^{46}$ Anhand einer Studie der

43 Vgl. ebenda, S. 14.

44 Belgien, Deutschland, Frankreich, Finnland, Italien, Niederlande, Portugal, Rumänien, Schweden und Slowenien. So wurde etwa in den Niederlanden die "gatekeeper“-Funktion des bis 2009 zentralen EU-Ausschusses des Senats (Eerste Kamer) „Commissie voor Europese Samenwerkingsorganisaties (ESO)“ geschwächt. Seitdem können alle Ausschüsse eine Subsidiaritätsprüfung veranlassen. Der radikale Fall dieses „Mainstreamings“ lässt sich im schwedischen Riksdag beobachten: Dort erwägt man den Europaauschuss einzustellen, um Doppelarbeit zu vermeiden. Wir danken Jonas Buche für diesen Hinweis.

45 Kees Van Kersbergen / Bertjan Verbeek, The Politics of International Norms: Subsidiarity and the Imperfect Competence Regime of the European Union, in: European Journal of International Relations, 13. Jg. (2007), H. 2, S. $217-238$.

46 Aaron Martin, The Principle of Subsidiarity and Institutional Predispositions: Do the European Parliament, the German Bundestag, and the Bavarian Landtag Define Subsidiarity Differently?, CAP Working Paper, München 2010. 
Forschungsabteilung des polnischen Sejms lassen sich vier Gruppen beziehungsweise „Welten der Subsidiarität” innerhalb nationaler Parlamente ausmachen ${ }^{47}$ : Eine erste Gruppe definiert Subsidiarität als ein dynamisches Prinzip, das in geringerem Umfang eine juristische Prüfkategorie als vielmehr den Ausdruck politischen Willens darstellt (britisches Oberhaus, französische Nationalversammlung, italienische Abgeordnetenkammer, Litauen, Slowakei). ${ }^{48}$ Eine zweite Gruppe versucht, Interpretationsspielräume möglichst einzuengen und in einer strikt juristischen Auslegung zugleich die Verfahrensdetails festzulegen. In Deutschland, der niederländischen zweiten Kammer und in Portugal wird somit zusätzlich die Anspruchsgrundlage geprüft. ${ }^{49}$ Eine dritte Gruppe folgt einer minimalistischen Lesart des Vertrages und subsumiert darunter ausschließlich supranationale Sachverhalte (tschechischer Senat, Finnland). ${ }^{50}$ Gerade aufgrund der begrenzten Einzelermächtigung der EU, könnten Subsidiaritätsverletzungen kaum eine Rolle spielen. Eine letzte Gruppe thematisiert Fragen der Subsidiarität bislang gar nicht (italienischer Senat, Spanien, Zypern, Bulgarien, Frankreich, beide Kammern in Belgien, Luxemburg). ${ }^{51}$

\subsection{Die Barroso-Initiative: Politischer Dialog und Testlauf für die Subsidiaritätskontrolle}

Tatsächlich hat die Europäische Kommission einiges dafür getan, die Effektivität des Frühwarnsystems lange vor Inkrafttreten des Lissabon-Vertrages zu gewährleisten. Ein Blick auf diese Bestrebungen kann erste Hinweise auf den späteren Umgang mit dem neuen Regelwerk liefern. Auf Initiative des Kommissionspräsidenten José Manuel Barroso hin wurden die Elemente der Subsidiaritätsprüfung durch nationale Parlamente auf informaler Basis und ohne vertragliche Grundlage getestet. Im Jahr 2006 in Gang gesetzt, sollte die so genannte „Barroso-Initiative“ den Akteuren dabei helfen, ihre gesetzgeberische Rolle aktiver wahrzunehmen. ${ }^{52}$ Diese Maßnahme schloss alle nationalen Kammern zu einem sehr frühen Zeitpunkt der Politikformulierung ein und leitete ihnen sämtliche EU-Arbeitsdokumente zu, ohne die bis dahin übliche Vermittlerrolle der jeweiligen nationalen Regierungen in Anspruch zu nehmen. Seit dem 1. September 2006 leitet das Generalsekretariat der Kommission somit den Parlamenten alle Entwürfe für Gesetzgebungsvorhaben, Konsultationsdokumente (Grün- und Weißbücher) sowie ihr jährliches Arbeitsprogramm auf direktem Wege zu. Im Gegenzug dieses „politischen Dialogs“53 können die nationalen Parlamente ihre Stellungnahmen ebenfalls direkt der Kommission zusenden und dabei ihre Regierungen umgehen. Zur Vereinfachung dieses Austauschprozesses ist die IPEX-Datenbank eingerichtet worden. Dieses Instrument erlaubt nationalen Parlamenten einen automatisierten Zugriff auf alle Kommissionsdokumente, die Möglichkeit, Stellungnahmen einzustellen und

47 Bureau of Research, a.a.O. (Fn. 31), S. 8-11.

48 Vgl. ebenda, S. 9.

49 Vgl. ebenda.

50 Vgl. ebenda, S. 9 f.

51 Vgl. ebenda, S. 11.

52 Vgl. Carmen Preising, Der Umgang der nationalen Parlamente mit den neuen Rechten des Vertrags von Lissabon - die Perspektive der Europäischen Kommission, in: Gabriele Abels / Annegret Eppler (Hrsg.), Auf dem Weg zum Mehrebenenparlamentarismus?, Baden-Baden 2011, S. 149 - 158.

53 Ebenda, S. $153 \mathrm{f}$. 
vorallem die Aktivitäten der anderen Kammern zu verfolgen. Das bedeutet zudem, dass Rat und Europäisches Parlament ebenfalls ihre Arbeitsdokumente den Kammern verfügbar machen und zugleich auf deren „begründete Stellungnahmen“ formal reagieren müssen. ${ }^{54}$

Über diesen technischen Aspekt hinaus erscheint die Frage interessanter, was die Kommission dazu bewog, die Barroso-Initiative ins Leben zu rufen: Weshalb sollte die Brüsseler Behörde Gefahr laufen, zumindest theoretisch die Parlamente zu stärken, deren Einfluss zu Lasten der etablierten Akteure im europäischen Entscheidungsprozess ginge? Aus einer weniger konfrontativen Perspektive kann die Initiative allerdings auch als ein Instrument legislativer Feinsteuerung (und damit „besserer Regulierung“) verstanden werden, das letztlich sowohl Effizienz als auch Legitimität der EU erhöhen könnte, indem es potenzielle Vetospieler in einem frühen Stadium der Entscheidungsfindung einbezieht. ${ }^{55}$ Hinzu kommt, dass die faktischen Möglichkeiten für nationale Gesetzgeber, Kommissionsvorhaben zu blockieren, äußerst begrenzt sind. Insofern profitiert die Kommission, ohne viel an Einflussverlust dabei zu riskieren.

Der „politische Dialog“ zwischen der Kommission und den nationalen Parlamenten hat sich seit seinem Beginn intensiviert: Bis Ende 2009, also vor dem offiziellen Inkrafttreten des Lissabon-Vertrages, erhielt die Kommission insgesamt 618 Stellungnahmen von 36 Kammern (von 40) ${ }^{56}$ aus 26 Mitgliedstaaten, mit einem klaren Aufwärtstrend. ${ }^{57}$ In der Logik dieses Dialogs nicht überraschend, bringen die Parlamente aber in der überwiegenden Mehrheit ihre Zustimmung zu Dokumenten der Kommission zum Ausdruck. Dies erklärt auch die herausgehobene Stellung Portugals: Mit insgesamt 131 Stellungnahmen zwischen 2006 und 2009 gehen mehr als 20 Prozent aller auf das Konto der Assembleia da República; allerdings kam dabei wohl auch die Herkunft des Kommissionspräsidenten als zusätzlich motivierender Faktor zum Tragen. ${ }^{58}$ Daneben sind insbesondere Zweite Kammern aktiv gewesen. Zusammengenommen haben die Senate Frankreichs, Italiens und Tschechiens, der Bundesrat und das britische Oberhauses rund 40 Prozent aller Stellungnahmen eingereicht. Ansonsten beteiligten sich nur noch das dänische und das schwedische Parlament mehr als 20-mal in vier Jahren. Seit Beginn der Barroso-Initiative hat die Zahl der eingegangenen Stellungnahmen kontinuierlich zugenommen ${ }^{59}$ : Im ersten Jahr 2006 waren es 53, ein Jahr später bereits mehr als doppelt so viele (115), 200 im Berichtsjahr 2008 und 2009 250; und

54 Um diese Prozedur zu vereinfachen, verwenden sowohl die Kommission als auch der Rat das Übertragungssystem E-Greffe, um Daten in die IPEX-Datenbank einzustellen.

55 Interview mit dem Generalsekretariat der Europäischen Kommission.

56 Von 27 Mitgliedstaaten verfügen 14 über ein parlamentarisches Einkammer- und 13 über ein Zweikammersystem, insgesamt also 40 gesetzgebende Kammern.

57 Vgl. Kommission der Europäischen Gemeinschaften, Jahresbericht 2009 über die Beziehungen zwischen der Europäischen Kommission und den nationalen Parlamenten, KOM (2010) 291 endgültig, Brüssel am 2. Juni 2010.

58 Üblicherweise wird dem portugiesischen Parlament eine schwache Kontrollfunktion zugesprochen. Vgl. Ana Fraga, The Parliament of Portugal: Loyal Scrutiny and Informal Influence, in: Andreas Maurer / Wolfgang Wessels (Hrsg.), a.a.O. (Fn. 27), S. 359 - 375.

59 Datenquellen: Kommission der Europäischen Gemeinschaften, Jahresbericht 2008 über die Beziehungen zwischen der Europäischen Kommission und den nationalen Parlamenten, KOM (2009) 343 endgültig, Brüssel am 7. Juli 2009; Kommission der Europäischen Gemeinschaften, a.a.O. (Fn. 57). 
im ersten vertraglich verankerten Jahr der Subsidiaritätskontrolle 2010 erreichten 387 Stellungnahmen die Kommission (siehe unten)..$^{60}$

$\mathrm{Zu}$ rund einem Fünftel aller Anfragen hat die Kommission Erwiderungen verfasst, dabei aber in der Mehrzahl ihre Position beibehalten und Ausführungen zur Reichweite des Vorschlags ergänzt. Dabei kreisten die meisten parlamentarischen Eingaben nicht um Fragen der Subsidiarität, sondern prüften darin auch materiell Aspekte der Binnenmarktfreiheiten, der Rechtsnatur, der Umwelt oder ökonomischer Zusammenhänge.

Die 250 Stellungnahmen des Jahres 2009 bezogen sich insgesamt auf 139 Kommissionsdokumente. ${ }^{61}$ Von diesen gab es nur zehn Vorschläge, die vier oder mehr Stellungnahmen nach sich zogen (ausgenommen jener, die noch unter dem COSAC-Testlauf stattfanden, siehe unten und Abbildung 2). Den größten Widerhall fand das Stockholm-Programm ${ }^{62}$ (acht Stellungnahmen), eine grenzüberschreitende Gesundheitsrichtlinie ${ }^{63}$ (sieben), eine Richtlinie zum Verbraucherschutz ${ }^{64}$ (sechs), Rahmenbeschlüsse gegen Menschenhandel ${ }^{65}$ (sechs), ein Vorschlag zur Verhinderung von Kindesmissbrauch ${ }^{66}$ (fünf) sowie das Grünbuch zum Verbraucherschutz. ${ }^{67}$ Betrachtet man allein für 2009 das parlamentarische Interesse entlang der zuständigen Generaldirektionen, so lag der Schwerpunkt deutlich auf dem Bereich Justiz, Freiheit und Sicherheit (83 Stellungnahmen, einschließlich jener, die zu den zwei COSAC-Testläufen des Jahres zählten). Der Bereich Gesundheit und Verbraucher zählte 38 (inklusive COSAC siehe oben), Verkehr und Energie 22, Bildung und Kultur 14, Umwelt 12, und auf Unternehmen entfielen insgesamt zehn Stellungnahmen.

\subsection{Subsidiaritätskontrolle unter dem Vertrag von Lissabon}

Das Subsidiaritäts-Protokoll des Lissabon-Vertrages hat den mit der Barroso-Initiative begonnenen politischen Dialog zwischen der Europäischen Kommission und den nationalen Parlamenten in eine juristische Form gegossen. Mit der im Februar 2010 neu gewählten Kommission begann offiziell der Frühwarnmechanismus der Subsidiaritätskontrolle (zwischen dem 1. Dezember 2009 und Februar 2010 wurden keine Entwürfe für Rechtsakte vorgelegt). Im ersten Jahr wurden insgesamt 82 solcher Entwürfe formal dem Prüfschema des Frühwarnmechanismus unterzogen. ${ }^{68}$ Die Kommission hat zu diesen prä-legislativen Dokumenten 211 Stellungnahmen erhalten, wovon 34 als „begründete Stellungnahmen“ nach Art. 6 einen Verstoß gegen das Subsidiaritätsprinzip geltend machten. Bislang ist aber weder der Gelbe- noch der Orange-Karte-Mechanismus ausgelöst worden. Betrachtet man nicht nur die parlamentarischen Reaktionen auf prä-legislative Dokumente, wie sie das

60 Vgl. Kommission der Europäischen Gemeinschaften, Jahresbericht 2010 über die Beziehungen zwischen der Europäischen Kommission und den nationalen Parlamenten, KOM (2011) 345 endgültig, Brüssel am 10. Juni 2011.

61 Vgl. Kommission der Europäischen Gemeinschaften, a.a.O. (Fn. 57), S. 3 ff.

62 Vgl. KOM (2009) 262.

63 Vgl. KOM (2008) 414.

64 Vgl. KOM (2008) 614.

65 Vgl. KOM (2009) 136.

66 Vgl. KOM (2009) 135.

67 Vgl. KOM (2008) 794.

68 Vgl. Kommission der Europäischen Gemeinschaften, a.a.O. (Fn. 60), S. 3. 
Subsidiaritätsprotokoll vorsieht, sondern bezieht sämtliche Stellungnahmen im Rahmen des fortlaufenden politischen Dialoges mit ein, erhielt die Kommission 2010 insgesamt 387 Stellungnahmen zu rund 170 verschiedenen Kommissionsdokumenten. ${ }^{69}$ Ähnlich wie im Jahr zuvor führte nur eine geringe Zahl an Vorschlägen zu Stellungnahmen von mehr als drei Mitgliedstaaten. Die zwei besonders kontrovers diskutierten Entwürfe waren die Richtlinie über Saisonarbeitnehmer ${ }^{70}$ (16 Stellungnahmen) und eine Richtlinie über Einlagensicherungssysteme ${ }^{71}$ (elf Stellungnahmen). Zum Vorschlag zur Förderung der Entwicklung des ländlichen Raumes ${ }^{72}$ gingen acht und zum Grünbuch zur Bürgerinitiative ${ }^{73}$ sieben Stellungnahmen ein. Drei weitere Kommissionsvorschläge erhielten ebenfalls sieben Stellungnahmen: der FRONTEX-Vorsto $\aleph^{74}$, die Initiativen zur landwirtschaftlichen Direktzahlung ${ }^{75}$ und die Vorschläge zur Lebensmittelunterstützung für Bedürftige in der Union. ${ }^{76}$

Ähnlich den Stellungnahmen des Vorjahres lag auch 2010 der Schwerpunkt auf den Bereichen Justiz und Inneres, Institutionelle Fragen, Binnenmarkt sowie Landwirtschaft. Auf diese vier Politikfelder allein entfielen rund 56 Prozent aller eingegangenen Stellungnahmen (siehe Abbildung 1).

Bei den schon genannten 2010 am stärksten diskutierten Entwürfen fielen neun der sechzehn Stellungnahmen zu der Richtlinie zu „Drittstaatsangehörigen in der Saisonarbeit“77 „begründet" ${ }^{\text {78 }}$ aus und machten damit einen Verstoß gegen das Subsidiaritätsprinzip geltend. ${ }^{79}$ Von den elf parlamentarischen Reaktionen auf die Neufassung des Richtlinienentwurfes zur Einlagensicherung waren fünf Subsidiaritätsrügen. ${ }^{80}$ Obwohl die vom Bundesrat

69 Vgl. ebenda, S. 6.

70 Vgl. KOM (2010) 379.

71 Vgl. KOM (2010) 368.

72 Vgl. KOM (2010) 537.

73 Vgl. KOM (2009) 622.

74 Vgl. KOM (2010) 61.

75 Vgl. KOM (2010) 539.

76 Vgl. KOM (2010) 486.

77 KOM (2010) 379; Ratsdokument 12208/10.

78 Österreich (beide Kammern), Tschechische Republik (beide Kammern) und die Niederlande (beide Kammern). Der polnische Senat und das britische Unterhaus fassten ihre Beschlüsse nicht im Plenum und die begründete Stellungnahme des britischen Oberhauses versäumte die Einreichungsfrist.

79 Der Europaauschuss des britischen Unterhauses formulierte am 13. Oktober 2010 folgendermaßen: „[...] the Treaty on the Functioning of the European Union does not empower the EU to determine how many labour migrants to admit. Decisions on the volumes of admissions remain in the hands of Member states. EU legislation on seasonal workers cannot alter that fact and so we do not accept the Commission's assertion that the draft Directive is crucial for securing effective co-operation with third countries"; http:/www.parliament.uk/documents/commons-committees/ european-scrutiny/13October2010.pdf (Abruf am 6. Juni 2012).

80 Deutscher Bundesrat, Bundestag, schwedischer Riksdag, dänischer Folketing und britisches Unterhaus. Dieser Kommissionsvorschlag führte zum erstmaligen Gebrauch der Subsidiaritätsrüge durch den Bundesrat und den Deutschen Bundestag. Vgl. Bundesrat, Vorschlag für eine Richtlinie des Europäischen Parlaments und des Rates über Einlagensicherungssysteme (Neufassung); KOM (2010) 368 endg.; Drs. 437/10 vom 24. September 2010; http://ec.europa.eu/dgs/secretariat_general/relations/relations_other/npo/docs/germany/2010/com20100368/com20100368_bundesrat_opinion_de.pdf (Abruf am 6. Juni 2012). Das britische Unterhaus hatte seine Stellungnahme nicht im Plenum gefasst und erst nach der Einreichfrist versandt, so dass je nach Quelle vier oder fünf Rügen gezählt werden. Vgl. COSAC, Sixteenth Bi-annual Report: Developments in Euro- 


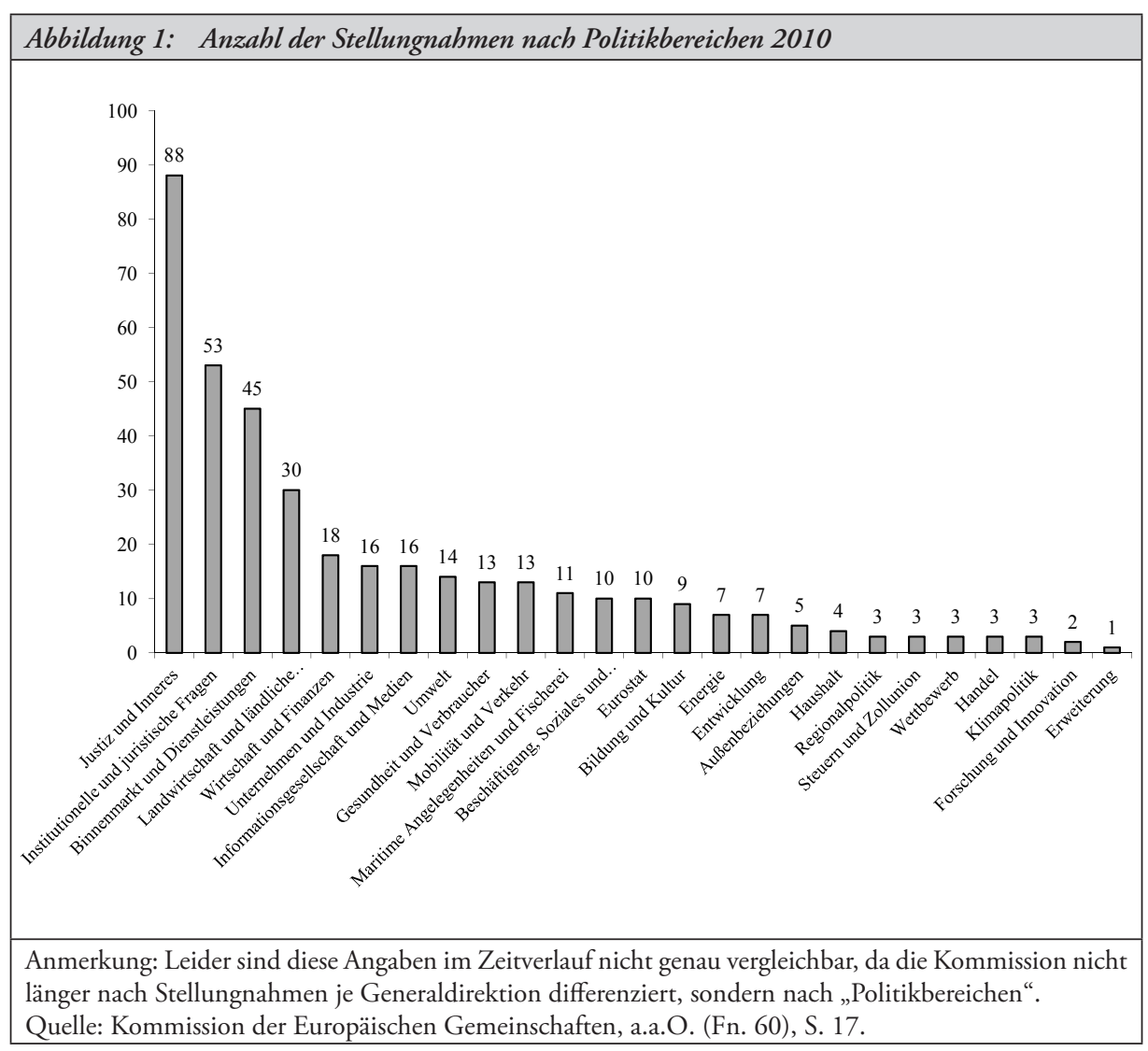

ausgehende Initiative letztlich das Quorum verfehlte, wurde damit immerhin deutlich, dass der Bundesrat seiner Aufsichtsrolle entsprechend nachkam. ${ }^{81}$ Bislang führten lediglich fünf Entwürfe für Rechtsakte zu mehr als zwei Subsidiaritätsrügen. Dabei zeigt im Vergleich ein unterschiedlicher Gebrauch der Stellungnahmen: Die portugiesische Volksvertretung hat wie seit Beginn der Barroso-Initiative mit Abstand die meisten eingereicht, allerdings die Rügemöglichkeit bislang nicht genutzt. Wie bereits 2009 waren auch 2010 Zweite Kammern besonders aktiv. Allein die Zweiten Kammern Italiens, Tschechiens, Deutschlands und Großbritanniens verantworteten rund 40 Prozent aller Stellungnahmen. Das Bild bei den Subsidiaritätsrügen sieht ähnlich aus: 14 der 34 Rügen sind von Zweiten Kammern eingereicht worden. Auffallend ist zudem, dass 2010 sechs Mitgliedstaaten weder allgemeine noch begründete Stellungnahmen abgegeben haben.

pean Union Procedures and Practices Relevant to Parliamentary Scrutiny, Prepared by the COSAC Secretariat and presented to: XLVI Conference of Parliamentary Committees for Union Affairs of Parliaments of the European Union, Warschau vom 2. bis 4. Oktober 2011.

81 So etwa der Vorsitzende des Europaausschusses des Deutschen Bundestages, Gunther Krichbaum; http://www.bundestag.de/dokumente/textarchiv/2010/32624033_kw49_lissabon/index.html (Abruf am 6. Juni 2012). 


\begin{tabular}{|l|l|c|c|c|l|c|c|}
\hline \multicolumn{7}{|c|}{ Tabelle 1: Anzahl der eingegangenen Stellungnahmen 2010} \\
\hline Land & \multicolumn{1}{|c|}{ Kammer } & Zahl & Rügen & Land & \multicolumn{1}{|c|}{ Kammer } & Zahl & Rügen \\
\hline PT & Assembleia da Republica & 106 & 0 & UK & House of Commons & 3 & 3 \\
\hline IT & Senato della Repubblica & 71 & 1 & NL & $\begin{array}{l}\text { Eerste Kamer Staten } \\
\text { Generaal }\end{array}$ & 3 & 0 \\
\hline CZ & Senát & 29 & 1 & PL & Sejm & 2 & 2 \\
\hline IT & Camera dei Deputati & 25 & 0 & NL & Beide Kammern & 2 & 2 \\
\hline DE & Bundesrat & 23 & 1 & RO & Beide Kammern & 2 & 0 \\
\hline SE & Riksdagen & 20 & 3 & NL & $\begin{array}{l}\text { Tweede Kamer Staten } \\
\text { Generaal }\end{array}$ & 1 & 0 \\
\hline AT & Bundesrat & 13 & 2 & CY & $\begin{array}{l}\text { House of Representati- } \\
\text { ves }\end{array}$ & 1 & 0 \\
\hline AT & Nationalrat & 12 & 1 & LV & Saeima & 1 & 0 \\
\hline UK & House of Lords & 12 & 2 & BE & $\begin{array}{l}\text { Chambre des Représen- } \\
\text { tants }\end{array}$ & 1 & 0 \\
\hline DK & Folketinget & 11 & 2 & FI & Eduskunta & 1 & 0 \\
\hline RO & Senatul & 9 & 0 & BE & Sénat & 0 & 0 \\
\hline LU & Chambre des Députés & 7 & 3 & BG & Narodno Sabrania & 0 & 0 \\
\hline DE & Deutscher Bundestag & 6 & 1 & EE & Riigikogu & 0 & 0 \\
\hline PL & Senat & 5 & 4 & FR & Assemblée Nationale & 0 & 0 \\
\hline GR & Abgeordnetenkammer & 4 & 0 & HU & Országgyúlés & 0 & 0 \\
\hline LT & Seimas & 4 & 2 & MT & Kamra tad-Deputati & 0 & 0 \\
\hline ES & $\begin{array}{l}\text { Congreso de los Diputa- } \\
\text { dos und Senado (beide } \\
\text { Kammern) }\end{array}$ & 4 & 0 & SK & Národná Rada & 0 & 0 \\
\hline IR & $\begin{array}{l}\text { Oireachtas (beide Kam- } \\
\text { mern) }\end{array}$ & 3 & 0 & SI & Državni svet & 0 & 0 \\
\hline CZ & Poslanecká sněmovna & 3 & 1 & SI & Državni zbor & 0 & 0 \\
\hline FR & Sénat & 3 & 3 & RO & Camera Deputaților & 0 & 0 \\
\hline $\begin{array}{l}\text { Anmerkung: Im niederländischen und rumänischen Fall kam es zu gemeinsamen Stellungnahmen beider } \\
\text { Qummelle: Kommission der Europäischen Gemeinschaften, a.a.O. (Fn. 60), S. 13 ff. }\end{array}$ & & \\
\hline
\end{tabular}

Der ansteigende Trend hielt auch 2011 an: Weitere 35 begründete Stellungnahmen sind bei der Kommission eingetroffen. ${ }^{82}$ Auch die Aktivität Zweiter Kammern hielt an, wobei der polnische Senat mit acht, der italienische mit vier, das niederländische Oberhaus sowie der französische Senat mit drei Subsidiaritätsrügen herausragen. Besonders kontrovers diskutiert wurde eine Initiative zur Vereinheitlichung der Bemessungsgrundlage bei Körperschaftssteu$e^{83}{ }^{83}$, die insgesamt neun Rügen erhielt. Wenig verwunderlich votierten insbesondere Kammern aus Mitgliedstaaten mit einem hohen beziehungsweise sehr niedrigen Steuersatz gegen diesen Richtlinienentwurf. ${ }^{84}$

82 Stand September 2011, vgl. COSAC, a.a.O. (Fn. 80), S. 27 ff. Konsolidierte Daten der Europäischen Kommission werden voraussichtlich ab Juli 2012 verfügbar sein.

83 Vgl. KOM (2011) 121.

84 Bulgarien, Niederlande (Tweede Kamer), Irland, Malta, Polen (Sejm), Rumänien, Slowakei, Schweden und Großbritannien. 
Nach wie vor bleibt aber die Nutzung der Subsidiaritätsrügen wie auch des politischen Dialogs sehr disparat. Dabei ist die Zahl der Stellungnahmen ein zweifelhafter Indikator für einen gestiegenen Einfluss der Parlamente. ${ }^{85}$ So hat etwa der Deutsche Bundestag bislang zwar nur eine geringe Anzahl von Stellungnahmen verabschiedet; dies weist aber eher darauf hin, dass andere Wege der Einflussnahme, etwa der direkte Austausch mit der Bundesregierung oder Informationsflüsse über das Brüsseler Büro des Bundestags, vielversprechender sind. ${ }^{86}$ Europapolitik bleibt aus Sicht der Mehrheitsfraktionen ein Balanceakt zwischen Kontrolle und Unterstützung und zwar besonders für Abgeordnete starker Kammern in wichtigen Mitgliedstaaten. ${ }^{87}$ Ein effektives EU-Monitoring spielt dabei gerade für Oppositionsparteien eine wichtige Rolle. Die ersten deutschen Erfahrungen belegen sowohl eine Zunahme an eigenen Entwürfen als auch überhaupt ein gestiegenes Interesse an europäischen Themen ${ }^{88}$, und dies trotz einer Oppositionslogik, wonach Initiativen in der Regel folgenlos bleiben müssen. Obwohl also die Zahl der Stellungnahmen noch nichts über den faktischen Einfluss einzelner Kammern aussagt, zeigt sich daran doch das Maß an politischem Bewusstsein für die Ausübung parlamentarischer Kontrolle in der Union.

Die bisher durchgeführten Subsidiaritätsprüfungen belegen zwei Erwartungen: Erstens scheinen die Acht-Wochen-Frist sowie das Mehrheitsquorum Schwierigkeiten zu machen. Immerhin 24 der 40 Kammern haben angegeben, dass diese Zeitspanne nicht ausreiche, um effektiv kontrollieren und insbesondere die Qualität der „impact assessments“ überprüfen zu können. ${ }^{89}$ Zweitens fällt auf, dass Parlamente in der Mehrzahl der eingereichten Stellungnahmen nicht allein den prozeduralen Gehalt des Frühwarnmechanismus aufgreifen und folglich nicht ausschließlich die Qualität der Subsidiaritätsbegründung der Kommission prüfen: Vielmehr wird zusätzlich die jeweilige Kompetenzgrundlage als erweiterter Prüfmaßstab $^{90}$ einbezogen. Bislang sahen 16 Kammern die aus ihrer Sicht falsche rechtliche Grundlage als ebenbürtigen Verstoß gegen das Subsidiaritäts-Protokoll an und reichten entsprechend begründete Stellungnahmen ein. ${ }^{91}$

\section{Subsidiaritätskontrolle als Problem kollektiven Handelns}

Die bisherigen Erkenntnisse machen bei der effektiven Einbeziehung nationaler Gesetzgebungskörperschaften zwei Schwierigkeiten deutlich: erstens das Schmieden inter-parlamentarischer Mehrheiten angesichts der noch neuen Kontrollinstrumente und der unterschied-

85 Vgl. Sebastian Gröning-von Thüna, Die neuen Begleitgesetze zum Vertrag von Lissabon aus Sicht des Deutschen Bundestages - offene Fragen und neue Herausforderungen, in: integration, 33. Jg. (2010), H. 4, S. 325 f.

86 Vgl. COSAC, a.a.O. (Fn. 80), S. 35.

87 Dies gilt nicht im selben Maße für kleinere Mitgliedstaaten: Dort sind Abgeordnete durch die geringere Verhandlungsmacht ihrer Regierungen zusätzlich eingeschränkt (eigene Interviews mit ungarischen Abgeordneten, siehe Fußnote 26).

88 Dies belegen eigene Interviews im Deutschen Bundestag, siehe Fußnote 26.

89 Vgl. COSAC, a.a.O. (Fn. 80), S. 7, S. 36 f.

90 Juristisch gerechtfertigt erscheint dies aber im Sinne einer „umfassenden Überprüfbarkeit der Schrankentrias des Art. 5 EUV[...]“. Christian Calliess / Matthias Ruffert, EUV/AEUV. Kommentar, München, 2011, Rn. 37.

91 Vgl. COSAC, a.a.O. (Fn. 80), S. 7, S. $38 \mathrm{ff}$. 
lichen Wichtigkeit europäischer Vorgaben im nationalen Kontext. Zweitens ist für den einzelnen Abgeordneten eine wirksame Kontrolle exekutiver Europapolitik ein komplexes Geflecht: Mitglieder der regierenden Koalitionsparteien werden ihrer Delegationsrolle entsprechend eher Vorstößen zustimmen, als diese übermäßig zu kontrollieren. Insofern wird man Voten parlamentarischer Ausschüsse kaum als blockierendes Entscheidungsinstrument beobachten können, da stabile Mehrheitspositionen nicht im Plenum oder im Ausschuss gefährdet werden..$^{92}$ Vielmehr werden vor allem Mitglieder der Opposition versuchen, jede verfahrenstechnische Gelegenheit auszuschöpfen, ihr Missfallen gegenüber der Regierung auszudrücken. ${ }^{93}$

Aus Sicht nationaler Parlamente lässt sich die Möglichkeit, gegenüber einem Legislativvorschlag der Kommission ein suspensives Veto einzulegen, als Kollektivgut begreifen ${ }^{94}$ : Das erforderliche einfache Mehrheitsquorum von 27 der 54 Stimmen im Sinne des Subsidiaritätskontrollmechanismus erhöht die relative Verhandlungsposition nationaler Parlamente innerhalb der europäischen Entscheidungsstrukturen. Allerdings gibt es insofern Anreize zum „Trittbrettfahren“, als es rational erscheint, auf hinreichend viele „begründete Stellungnahmen" der jeweils anderen Kammern zu vertrauen - und das selbst im idealtypischen Fall gleicher Präferenzen gegenüber einem Rechtsaktentwurf. Daraus ergibt sich, dass es Promotoren des Frühwarnmechanismus sehr schwer fallen dürfte, andere Kammern zu kollektivem Handeln zu bewegen.

Um solche Mehrheiten schmieden und somit den Frühwarnmechanismus effektiv einsetzen zu können, sind die nationalen Parlamente auf einen funktionierenden Informationsaustausch und länderübergreifende Zusammenarbeit angewiesen. Neue technische Hilfsmittel wie die IPEX-Datenbank, die Zusammenarbeit mit der COSAC sowie die Kontakte zwischen den Verbindungsbüros in Brüssel haben diese horizontale Kooperation in jüngster Zeit erleichtert. Auf diese Weise erfolgte auch ein Austausch hinsichtlich „best practices" der exekutiv-legislativen Zusammenarbeit. ${ }^{95}$ Dennoch dürfte es schwierig bleiben, das erforderliche Quorum zu erzielen: angesichts unterschiedlicher parlamentarischer Beteiligungen, nationaler Subsidiaritätskonzepte und nicht zuletzt aufgrund der Neuartigkeit des Instruments.

Um zumindest einen Teil dieser erwarteten Hindernisse aus dem Weg zu räumen, waren vor Inkrafttreten des Lissabon-Vertrages Subsidiaritätstestläufe (siehe Abbildung 2) vereinbart worden. Dieses von der COSAC organisierte Abstimmungsverfahren suchte eben jenem Kollektivgut-Problem entgegenzutreten. Zwischen 2005 und 2009 wurden Testläufe zu acht

92 Vgl. Philipp Kiiver, German Participation in EU Decision-Making after the Lisbon Case: A Comparative View on Domestic Parliamentary Clearance Procedures, in: German Law Journal, 10. Jg. (2009), H. 8, S. 1293; Ronald Holzhacker, The Power of Opposition Parliamentary Party Groups in European Scrutiny, in: Journal of Legislative Studies, 11. Jg. (2005), H. 3/4, S. 428 - 445.

93 Deshalb wurde das Klagerecht vor dem EuGH als Minderheitsrecht ausgeführt: 25 Prozent der Mitglieder des Bundestages oder der Stimmen des Bundesrates sind hinreichend. Vgl. Thomas Silberhorn, Zur Rolle des Deutschen Bundestages nach dem Lissabon-Urteil des Bundesverfassungsgerichts, in: Ralf Thomas Baus / Michael Borchard / Günter Krings (Hrsg.), Europäische Integration und deutsche Verfassungsidentität, St. Augustin / Berlin 2010, S. 137 - 146.

94 Vgl. Mancur Olson, The Logic of Collective Action: Public Goods and the Theory of Groups, Cambridge 1965.

95 Vgl. Copenhagen Parliamentary Guidelines. Guidelines for Relations between Governments and Parliaments on Community Issues (Instructive Minimum Standards), COSAC, 27. Januar 2003;

OJ C154/1, 2. Juli 2003. 


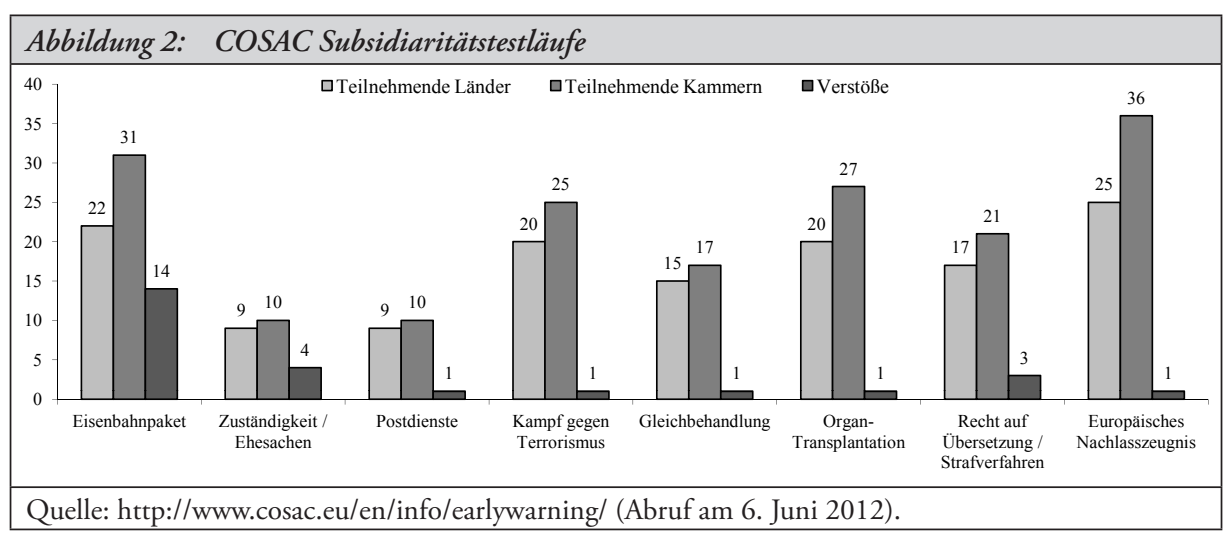

Legislativvorschlägen unter Federführung der COSAC durchgeführt. ${ }^{96}$ Lag die anfängliche Teilnahmequote noch sehr hoch, fiel sie 2006 und 2007 leicht ab und mit ihr die Zahl der beanstandeten Subsidiaritätsverstöße. Im Rahmen der Lissabon-Vorbereitungen nahm die Zahl der teilnehmenden Kammern 2009 wieder zu. Der letzte Testlauf wurde aus technischen Gründen bereits nach Maßgabe der neuen Rechtslage vollzogen.

Die Testläufe offenbarten einige interessante Defizite: Wie befürchtet, monierten Angehörige der Parlamentsverwaltungen, dass die Acht-Wochen-Frist für eine umfassende Prüfung zu kurz ausfalle, insbesondere während der Urlaubsmonate. ${ }^{97}$ Ein weiteres Problem lag darin, dass nicht alle Dokumente in allen Amtssprachen vorlagen. Hinzu kam, dass der Ablauf der Mehrheitsbildung informelle Abstimmungen zwischen den Kammern vor dem Einreichen einer begründeten Stellungnahme erforderte. Die parlamentarischen Konsultationsprozesse dabei länderübergreifend in Einklang zu bringen, erwies sich als schwierig. Gleichwohl wurden auf diese Weise im „laufenden Betrieb“ Lernprozesse zwischen der Kommission und Parlamentsverwaltungen angestoßen.

Das Gesamtbild zeigte nach wie vor ungelöste Abstimmungsprobleme sowohl auf nationaler Ebene (zwischen Legislative und Exekutive, aber auch in Zweikammersystemen zwischen den Häusern) als auch zwischen den Parlamenten der Mitgliedstaaten. Einmal mehr wurden in den Testläufen die unterschiedlichen Kontrollarrangements gegenüber den Exekutiven deutlich. ${ }^{98}$ Dies dürfte vor allen Dingen in der oben bereits erwähnten vielfältigen Konzeption des Strukturprinzips Subsidiarität begründet sein, die auch auf seine institutionelle Verankerung durchschlägt. Tatsächlich führen nur wenige Parlamente eine systematische Überprüfung auf Vereinbarkeit mit Subsidiarität durch. Während in einigen die Europaausschüsse in Vertretung des Plenums entscheiden dürfen, ist in anderen dessen Zustimmung notwendig. In einer weiteren Ländergruppe müssen die Positionen der Fachausschüsse berücksichtigt werden. Dasselbe gilt für Mitgliedstaaten mit regionalen Parlamenten. Diese disparaten nationalen Varianten parlamentarischer Kontrollkultur können

$96 \mathrm{Zu}$ den acht Subsidiaritätstestläufen siehe im Einzelnen: http://www.cosac.eu/en/info/earlywarning/ (Abruf am 6. Juni 2012)

97 Aus diesem Grund klammerte die Kommission den Monat August offiziell aus der Acht-WochenRegelung aus.

98 Vgl. COSAC, a.a.O. (Fn. 41). 
dazu führen, dass es schlicht zu lange dauert, eine kritische Masse an Kammern für den Frühwarnmechanismus zu aktivieren.

Die COSAC hat 2010 trotz der stattgefundenen Lernprozesse beschlossen, die Testläufe einzustellen und damit diese länderübergreifende Koordination etwas loser zu knüpfen. Obgleich sämtliche Parlamente deren Notwendigkeit anerkennen, scheint doch die genaue Vorstellung darüber zu fehlen, wie diese in der Praxis ausgestaltet sein sollte. So verspricht etwa die bereits erwähnte IPEX-Datenbank, die von der COSAC und Berichterstattern in den nationalen Parlamentsverwaltungen unterhalten wird, den Austausch über MonitoringAspekte zu fördern. Zwar hat das System seine Vorzüge, der Gebrauch ist aber nur punktuell überzeugend; auf manche Information erfolgt der Zugriff zu spät. Das hängt auch damit zusammen, dass mitunter auf Parlamentsseite Ressourcen fehlen, um aktuelle Informationen einzupflegen, und zudem ihr Nutzen als eingeschränkt wahrgenommen wird ${ }^{99}$ - sprich, eine stärkere formale und institutionalisierte Zusammenarbeit bietet zwar Vorteile, ist aber kostenintensiv. Deshalb betonen Stäbe in Parlamentsverwaltungen gegenwärtig vor allem die Bedeutung informeller Kontakte auf Mitarbeiterebene. Wichtiger werden in diesem Zusammenhang die ständigen Vertreter der Parlamente, deren Büros sich im Europäischen Parlament auf einem Stockwerk befinden. Dabei bieten auch ihre regelmäßigen Montagtreffen (Monday Morning Meeting) ein Forum zum Informationsaustausch und zur frühzeitigen Sondierung der Positionen der anderen Parlamente. Diese Form der Repräsentation ist in den vergangenen Jahren intensiviert worden: 2011 verzichteten lediglich Malta und die Slowakei auf ein eigenes parlamentarisches Büro bei der EU.

\section{Rollen und Selbstwahrnehmungen nationaler Abgeordneter}

Allerdings birgt die Fokussierung auf den weiteren Ausbau formaler parlamentarischer Kontrollrechte und deren politisch mühsame und zeitaufwendige Anwendung die Gefahr, einen wesentlichen Faktor zu übersehen. Die nachhaltige Wirkung solcher gutgemeinter Initiativen verliert sich allzu rasch, wenn es den einzelnen Abgeordneten an Anreizen fehlt, von diesen neuen Rechten auch tatsächlich Gebrauch zu machen. Bislang konnten von den Veränderungen des Lissabon-Vertrages und der verbesserten interparlamentarischen Zusammenarbeit die Parlamentsverwaltungen eher profitieren als die eigentlichen Adressaten, die Parlamentarier. ${ }^{100}$ Zumindest zum Teil ist dies eine Folge der beschränkten Zeit- und Personalkapazitäten der Abgeordneten. So zeigen 2010 und 2011 durchgeführte Interviews mit Abgeordneten in mehreren Parlamenten, dass ihrer tatsächlichen Mitarbeit im parlamentarischen Mehrebenensystem der EU enge Schranken gesetzt sind ${ }^{101}$; besonders problematisch scheint die parlamentarische Ressourcenausstattung zudem in den mittel- und osteuropäischen Mitgliedstaaten zu sein. ${ }^{102}$

99 Eigene Interviews, Ungarisches Parlament, Dezember 2009.

100 Wir danken Thomas Christiansen für diesen Hinweis.

101 Vgl. Sabine Kropp / Jonas Buche / Aron Buzogány, Parlamentarisch-exekutive Steuerung in europäisierten Fachpolitiken - ein Blick auf die Mikroebene, in: Gabriele Abels / Annegret Eppler (Hrsg.), Auf dem Weg zum Mehrebenenparlamentarismus?, Baden-Baden 2011, S. 227 - 239.

102 Vgl. Viera Knutelská, Working Practices Winning Out over Formal Rules: Parliamentary Scrutiny of EU Matters in the Czech Republic, Poland and Slovakia, in: Perspectives on European Politics and Society, 12. Jg. (2011), H. 3, S. 320 - 339; Aron Buzogány, True Control or Trompe-l'œil? 
Noch wichtiger sind in diesem Zusammenhang vielleicht die Rollenverständnisse ${ }^{103}$ und Selbstwahrnehmungen der Abgeordneten, die, wie in einer länderübergreifenden Studie von Bernhard Weßels nachgewiesen, eher schwach europäisiert sind. ${ }^{104}$ Daraus folgt fast schon zwangsweise, dass die der Regierung mühsam abgetrotzten Rechte kaum genutzt werden. Im deutschen Kontext spielen dabei auch das Wahlrecht und die daraus abgeleitete Unterscheidung zwischen „Wahlkreis-“ und „Listenabgeordneten“ eine nicht zu vernachlässigende Rolle. ${ }^{105}$ Zwar ist unter MdBs die Rollenorientierung mit einem Wahlkreisfokus insgesamt stärker ausgeprägt als die Selbstwahrnehmung als „Parteisoldat“106. Doch es überrascht kaum, dass insbesondere die vor allem im Wahlkreis aktiven „Generalisten“ oft weder die notwendige Zeit noch die Expertise mitbringen, die für die Mitgestaltung oder die parlamentarische Kontrolle von EU-Vorlagen notwendig ist. Gleichwohl scheint die 2010 einsetzende Krise der Europäischen Währungsunion die Bedeutung europäischer Politik zu steigern und zu Rollenkonflikten unter den Abgeordneten zu führen, da diese befürchten müssen, ihre fehlende EU-Expertise könne sich als Nachteil für ihre Wiederwahlchancen auswirken.

Als Folge scheint die Notwendigkeit, die europäische Ebene als festen Bestandteil im eigenen Rollenverständnis zu verankern, von nationalen Abgeordneten zunehmend erkannt zu werden; so geht Sabine Kropp davon aus, dass sich mit dem „europäischen Experten“ zunehmend ein neues Rollenverständnis auch jenseits des Europaauschusses herausbildet. Abgeordnete, die sowohl fachpolitische Expertise als auch prozedurales Wissen über das Mehrebenensystem mitbringen, sind in der Lage, neben der Aufrechterhaltung von Kontakten auf der nationalen Ebene Netzwerke mit einer Vielzahl von Akteuren in den europäischen Institutionen zu unterhalten. ${ }^{107}$ Daher räumen, zumindest im deutschen Fall, Abgeordnete durchaus ein, sich stärker um „die europäische Dimension ihres Fachgebiets kümmern zu müssen"108, gerade auch wegen des anerkannt uneinholbaren Wissensvorsprungs der Ministerialbürokratie.

Parliamentary Scrutiny of EU Policies in the Hungarian Parliament, in: Krisztina Arató / Zsolt Enyedi / Ágnes Lux (Hrsg.), Structures and Futures of Europe, Budapest 2012, S. 129 - 153.

$103 \mathrm{Zu}$ Rollentheorie in Parlaments- und Abgeordnetenstudien siehe insbesondere Thomas Saalfeld I Wolfgang C. Müller, Roles in Legislative Studies: A Theoretical Introduction, in: The Journal of Legislative Studies, 3. Jg. (1997), H. 1, S. 1 - 16; Sabine Kropp / Matthias Ruschke, Parlament und Verwaltung: in Rollenpartnerschaft vereint? Ein Plädoyer für die Verknüpfung von Parlaments- und Verwaltungsforschung, in: ZParl, 41. Jg. (2010), H. 3, S. $638-660$.

$104 \mathrm{Vgl}$. Bernhard Weßels, Roles and Orientations of Members of Parliament in the EU Context: Congruence or Difference? Europeanisation or not?, in: The Journal of Legislative Studies, 11. Jg. (2005), H. 3, S. $446-465$.

$105 \mathrm{Vgl}$. Werner J. Patzelt, German MPs and their Roles, in: The Journal of Legislative Studies, 3. Jg. (1997), H. 1, S. $55-78$.

106 Sabine Kropp, German Parliamentary Party Groups in Europeanised Policymaking - Awakening from the Sleep? Institutions and Heuristics as MPs' Resources, in: German Politics, 19. Jg. (2010), H. 2, S. $123-147$.

107 Vgl. ebenda.

108 Sabine Kropp, Die Ministerialbürokratie als Rollenpartner der Fachpolitiker in den Fraktionen - auf nationaler Ebene und im Mehrebenensystem der EU. Ergebnisse einer Studie über den Deutschen Bundestag, in: Die Öffentliche Verwaltung, 63. Jg. (2010), H. 10, S. 413 - 422, S. 418. 


\section{Schlussfolgerung: Keine Lösung aller Probleme}

Mehr als zwei Jahre nach Inkrafttreten des Lissabon-Vertrages liegt es nahe zu prüfen, was aus der guten Absicht geworden ist, das Demokratiedefizit der Union durch eine Stärkung der nationalen Legislativen zu verringern. Lässt sich tatsächlich ein wesentlicher Unterschied im Alltag europäischer Gesetzgebung beobachten? Sind die neuen Schranken der Subsidiarität und die den Parlamenten gewährten Befugnisse mehr als unwirksame Berichtsverfahren für Abgeordnete, denen es nicht allein an Ressourcen fehlt, sondern teils auch an Expertise sowie hinreichenden Anreizen?

Zurzeit stehen die gestärkten Kontroll- und Mitsprachemechanismen einer Vielzahl an mitgliedstaatlichen Lösungen gegenüber. Unterschiede zeigen sich etwa im Gebrauch bindender Mandate, der aktiven Rolle Zweiter Kammern und insbesondere in vielfältigen Interpretationen des Subsidiaritätsgedankens. ${ }^{109}$ Doch nicht allein die Interpretationen variieren, sondern auch die Wertschätzung, die Parlamentarier dem europäischen Strukturprinzip „Subsidiarität“ insgesamt beimessen. Während einige Kammern an Entwürfen für Rechtsetzungsakte lediglich die ausreichende Subsidiaritätsbegründung der Kommission prüfen, erweitern andere die Prüfung auch auf die dazugehörige Kompetenzgrundlage. Das novellierte Subsidiaritätsprotokoll erschwert noch die Suche nach der adäquaten Lesart: In der ursprünglichen Fassung des Amsterdamer Vertrages lag ein Subsidiaritätsverstoß erst dann vor, wenn der Sachverhalt „transnationale Aspekte“ aufwies. ${ }^{110}$ Insofern werden feine Grenzverletzungen der Subsidiarität und der Verhältnismäßigkeit umstritten bleiben - zumindest solange nicht künftige EuGH-Entscheidungen zu Subsidiaritätsklagen ${ }^{111}$ dazu beitragen werden, Interpretationsspielräume einzuengen und Prüfkriterien zu präzisieren.

Es ist unverkennbar, dass der Frühwarnmechanismus noch in den Anfängen steckt. Bislang ist es zu keiner erfolgreichen, also kollektiven Subsidiaritätsrüge gekommen. Die kurze Frist von acht Wochen führt weiterhin zu Fragen, wie sich der länderübergreifende Informationsfluss (und damit die Mehrheitsbeschaffung) verbessern ließe. ${ }^{112}$ Rein technisch betrachtet, zielt die Formel „begründete Stellungnahme“ des Protokolls nur auf Widersprüche

109 Vgl. COSAC, Report on the Results of the Subsidiarity Check on the Proposal for a Directive of the European Parliament and of the Council on Standards of Quality and Safety of Human Organs Intended for Transplantation. Prepared by the COSAC Secretariat and presented to: XLI Conference of Community and European Affairs Committees of Parliaments of the European Union, Prag vom 10. bis 12. Mai 2009, S. 14.

110 Vgl. Subsidiaritäts-Protokoll Art. 5, Abs. 2, zitiert nach Jessica Koch / Matthias Kullas, Subsidiarität nach Lissabon - Scharfes Schwert oder stumpfe Klinge? cepStudie, März 2010, S. 6, S. 17 , http://www.cep.eu/fileadmin/user_upload/Kurzanalysen/Subsidiaritaet/Studie_Subsidiaritaet.pdf (Abruf am 10. Februar 2011). Aus Sicht der Kommission ist es aber ein Fortschritt, nicht mehr den „Anwendungsmodus, sondern Verfahrensaspekte“ zu prüfen. Kommission der Europäischen Gemeinschaften, Bericht der Kommission über die Anwendung der Grundsätze der Subsidiarität und Verhältnismäßigkeit (18. Bericht über „bessere Rechtsetzung“ 2010), KOM (2011) 344 endgültig, Brüssel vom 10. Juni 2011, S. 2.

111 Subsidiaritäts-Protokoll Art. 8, Abs. 1.

112 Gleichwohl sind diese hohen Hürden gewissermaßen eine bewusst in Kauf genommene „institutionelle Schwäche": Im Verfassungskonvent war kurze Zeit ein de facto Vetorecht für nationale Parlamente erwogen, aber später verworfen worden. So der ehemalige Präsident des Europäischen Parlaments Klaus Hänsch, Forum Subsidiaritätskontrolle, Berlin am 18./19. Oktober 2010. 
und Einwände und meint damit negative Einschätzungen ${ }^{113}$ europäischer Vorgaben. Indes, nur auf diesen „Watchdog“-Teil der Subsidiaritätskontrolle zu blicken, lässt einen wichtigen Aspekt außer Acht: Mitsprache per se und damit auch die Option Zustimmung äußern zu können, scheint, wie die Erfahrungen aus dem politischen Dialog mit der Kommission zeigen, für nationale Parlamente ebenfalls relevant zu sein. ${ }^{114}$

Schon jetzt zeigen sich jedoch einige strukturelle Schwierigkeiten im Zusammenhang mit der ex-ante Kontrolle und dem Subsidiaritäts-Monitoring. Trotz der Anreize, mittels des COSAC-Rahmenwerks institutionelles Lernen zu ermöglichen, offenbart sich das Auslösen des Gelbe-Karte-Mechanismus auch als ein Problem kollektiven Handelns. Allerdings ist das „Kollektivgut" eines suspensiven Vetos der nationalen Kammern schwierig zu realisieren, wenn die Mehrheitsbeschaffung eines Vorreiterstaates kostspielig ist. Dabei erschwert die national stark divergierende Auslegung des Subsidiaritätsgedankens diese Mehrheitsbeschaffung noch zusätzlich.

Hinzu kommen weitere Hindernisse seitens der Abgeordneten, die dazu beitragen, dass ihr Interesse an Subsidiaritätskontrolle begrenzt bleibt. Auch wenn sich dies zurzeit langsam zu ändern scheint, sind weder das Ausmaß an europarechtlicher Expertise noch die innenpolitische Bedeutung hinreichend groß, um Anreize für eine stärkere parlamentarische Kontrolle zu schaffen.

Zusammengenommen zeigt sich, dass die neuen Verfahren der Subsidiaritätskontrolle wie sie der Lissabon-Vertrag vorsieht (mitsamt der Klagemöglichkeit vor dem EuGH), in ihrer Praxis noch in den Anfängen stecken und Entwicklungsmöglichkeiten bieten. Sie sind weder nutzloses „Wollknäuel“ zum Zwecke parlamentarischer Beschäftigung noch die „Lösung aller Probleme " 115 hinsichtlich potenzieller Subsidiaritätskonflikte. In jedem Fall erfordert der Umgang mit ihnen für die Gesamtheit der nationalen Parlamente die Fähigkeit, kollektiv zu handeln. Ob nun mittelfristig in der Rolle einer „Dritten Kammer“ oder nicht: die 40 nationalen Gesetzgebungsorgane in der Europäischen Union sollten sich darüber klar werden, dass ihre Mitsprachemöglichkeiten gestiegen sind und damit zugleich ihre Fähigkeit, die europäische Öffentlichkeit zu erreichen. ${ }^{116}$

113 „Die nationalen Parlamente oder die Kammern eines dieser Parlamente können binnen acht Wochen nach dem Zeitpunkt der Übermittlung eines Entwurfs eines Gesetzgebungsakts in den Amtssprachen der Union in einer begründeten Stellungnahme an die Präsidenten des Europäischen Parlaments, des Rates und der Kommission darlegen, weshalb der Entwurf ihres Erachtens nicht mit dem Subsidiaritätsprinzip vereinbar ist. Dabei obliegt es dem jeweiligen nationalen Parlament oder der jeweiligen Kammer eines nationalen Parlaments, gegebenenfalls die regionalen Parlamente mit Gesetzgebungsbefugnissen zu konsultieren.“ Art. 6, Abs. 1 (SubsidiaritätsProtokoll).

114 Obwohl die faktischen Mitspracherechte begrenzt bleiben, könnte doch bereits die Existenz eines „politischen Dialogs" mit der Europäischen Kommission in der Folge zu einer besseren Folgenabschätzung und einer verbesserten ex ante Berücksichtigung von Subsidiaritätsbedenken führen.

115 Franz C. Mayer, Expertengespräch im Rechtsausschuss, Unterausschuss Europarecht des Deutschen Bundestages, Wortprotokoll, Berlin am 16. Juni 2010, S. 11; http://www.bundestag. de/bundestag/ausschuesse17/a06/ua_europarecht/expertengespraech_aktuell/Wortprotokoll.pdf (Abruf am 6. Juni 2012).

116 Eine stärker länderübergreifende und themenorientierte parlamentarische Zusammenarbeit fordert zum Beispiel Volker Kauder, Mehr Macht den Parlamenten, in: Financial Times Deutschland vom 14. Februar 2011, S. 24. 